Supporting Information

\title{
How Hydrogen and Oxygen Vapor Affect the Tribochemistry of Silicon- and Oxygen-Containing Hydrogenated Amorphous Carbon under Low Friction Conditions: A Study Combining X-Ray Absorption
} Spectromicroscopy and Data Science Methods

Filippo Mangolini ${ }^{1,2, *}$, Komlavi D. Koshigan ${ }^{3}$, Mark H. Van Benthem ${ }^{4}$, James A. Ohlhausen ${ }^{4}$, John B. McClimon ${ }^{5}$, James Hilbert ${ }^{6}$, Julien Fontaine ${ }^{3}$, Robert W. Carpick ${ }^{6}$

${ }^{1}$ Texas Materials Institute, The University of Texas at Austin, Austin, Texas 78712, USA

${ }^{2}$ Walker Department of Mechanical Engineering, The University of Texas at Austin, Austin, Texas 78712, USA

${ }^{3}$ Laboratoire de Tribologie et Dynamique des Systèmes, Ecole Centrale de Lyon, CNRS UMR 5513, Université de Lyon, 69134, Ecully cedex, France

${ }^{4}$ Sandia National Laboratories, Albuquerque, New Mexico 87185, USA

${ }^{5}$ Department of Materials Science and Engineering, University of Pennsylvania, Philadelphia, Pennsylvania 19104, USA

${ }^{6}$ Department of Mechanical Engineering and Applied Mechanics, University of Pennsylvania, Philadelphia, Pennsylvania 19104, USA

* Author to whom correspondence should be addressed. Electronic email: filippo.mangolini@austin.utexas.edu 


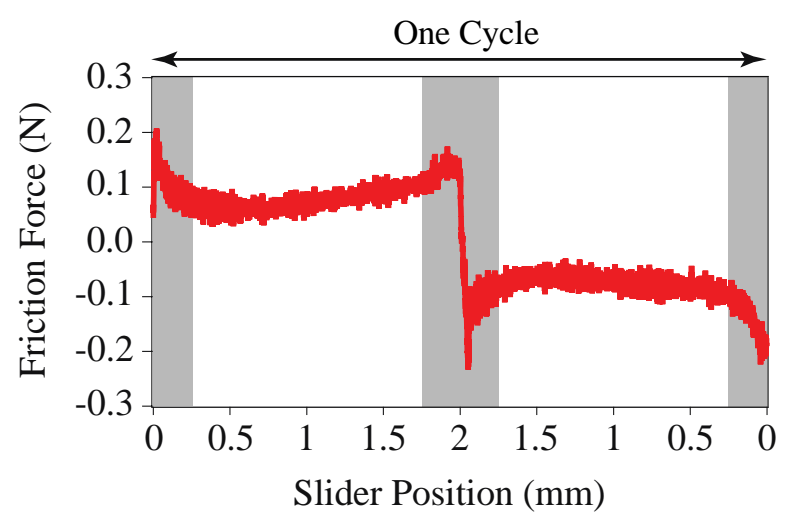

Figure S1. Friction force as a function of slider position during one reciprocating cycle (applied normal load: $3 \mathrm{~N}$ ). To calculate the average coefficient of friction in each cycle together with the corresponding standard deviation, only the data points corresponding to a lateral slider position between $1 / 8$ and $7 / 8$ of the stroke length were considered to avoid end-points effects. 